\title{
Strong room-temperature optical and spin polarization in InAs/GaAs quantum dot structures
}

\author{
Jan Beyer, Irina A Buyanova, S. Suraprapapich, C. W. Tu and Weimin Chen
}

\section{Linköping University Post Print}

N.B.: When citing this work, cite the original article.

Original Publication:

Jan Beyer, Irina A Buyanova, S. Suraprapapich, C. W. Tu and Weimin Chen, Strong roomtemperature optical and spin polarization in InAs/GaAs quantum dot structures, 2011, Applied Physics Letters, (98), 20, 203110.

http://dx.doi.org/10.1063/1.3592572

Copyright: American Institute of Physics http://www.aip.org/

Postprint available at: Linköping University Electronic Press http://urn.kb.se/resolve?urn=urn:nbn:se:liu:diva-68961 


\title{
Strong room-temperature optical and spin polarization in InAs/GaAs quantum dot structures
}

\author{
J. Beyer, ${ }^{1, a)}$ I. A. Buyanova, ${ }^{1}$ S. Suraprapapich, ${ }^{2}$ C. W. Tu, ${ }^{2}$ and W. M. Chen ${ }^{1, a)}$ \\ ${ }^{1}$ Department of Physics, Chemistry and Biology, Linköping University, SE-581 83 Linköping, Sweden \\ ${ }^{2}$ Department of Electrical and Computer Engineering, University of California, La Jolla, CA 92093, USA
}

(Received 27 January 2011; accepted 28 April 2011; published online 19 May 2011)

\begin{abstract}
Room-temperature optical and spin polarization up to $35 \%$ is reported in InAs/GaAs quantum dots in zero magnetic field under optical spin injection using continuous-wave optical orientation spectroscopy. The observed strong spin polarization is suggested to be facilitated by a shortened trion lifetime, which constrains electron spin relaxation. Our finding provides experimental demonstration of the highly anticipated capability of semiconductor quantum dots as highly polarized spin/light sources and efficient spin detectors, with efficiency greater than $35 \%$ in the studied quantum dots. (C) 2011 American Institute of Physics. [doi:10.1063/1.3592572]
\end{abstract}

Confinement of carriers in three dimensions in semiconductor quantum dots (QDs) enables both the study of a wealth of interesting physics in nanostructures and the vision of applications in nanoelectronic and nanophotonic devices. Due to the restraint in carrier motion, common spin relaxation processes connected with spin-orbit interaction that usually dominate in bulk and two-dimensional semiconductors, are expected to be strongly suppressed. ${ }^{1,2}$ This has led to the proposition of QD spins for applications in nanospintronics and quantum information technology. ${ }^{3,4}$ Thanks to the quantum confinement and the resulting stronger overlap of wave functions between recombining electrons and holes, QDs are also known to be efficient light emitters. They are therefore expected to hold great potential for highly efficient optical spin detectors as well as strongly polarized spin and light sources, providing opportunities for integrating spintronic and photonic functionality of QD systems. These expectations have been reinforced by numerous experimental confirmations of long spin relaxation times reported for carriers in the ground state of a QD at low temperatures., ${ }^{5,6}$ Unfortunately, experimental investigations of spin properties of QDs at room temperature (RT), relevant to practical device applications, still remain sparse. Reported values of RT electron spin polarization, by monitoring optical polarization in continuous-wave (cw) optical orientation or electrical injection experiments, have so far been largely limited to just a few percent. ${ }^{7,8}$ The highly anticipated potentials of QDs for RT spintronics have yet to be demonstrated.

In this work, we carry out a detailed investigation of spin injection and spin detection in self-assembled InAs/GaAs QDs at elevated temperatures up to RT using cw optical orientation spectroscopy. We are able to achieve record-high RT spin polarization (up to 35\%) in the QDs upon optical spin injection, demonstrating the capability of the QDs as efficient optical spin detectors and strongly polarized spin/light sources at RT.

The studied self-assembled InAs QD structures were grown by gas source molecular beam epitaxy on (001) semiinsulating GaAs substrates. ${ }^{9,10}$ A sketch of the sample structures is given in the inset of Fig. 1. The QDs studied by photoluminescence (PL) are sandwiched between a $300 \mathrm{~nm}$

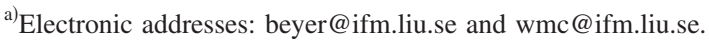

GaAs buffer layer and a $150 \mathrm{~nm}$ GaAs cover layer. The growth of each structure was finished by another layer of similar QDs for atomic force microscopy (AFM) studies. From AFM, the QDs are estimated to be typically about $2.5-11 \mathrm{~nm}$ in height and around $20-50 \mathrm{~nm}$ in diameter, with a density in the range of about $6 \times 10^{9} \mathrm{~cm}^{-2}$. This density is in the same order of magnitude as the sheet hole concentration provided by unintentional p-type doping due to residual carbon acceptors (i.e., $\mathrm{C}_{\mathrm{As}}$ ) in the GaAs barrier. As a result,

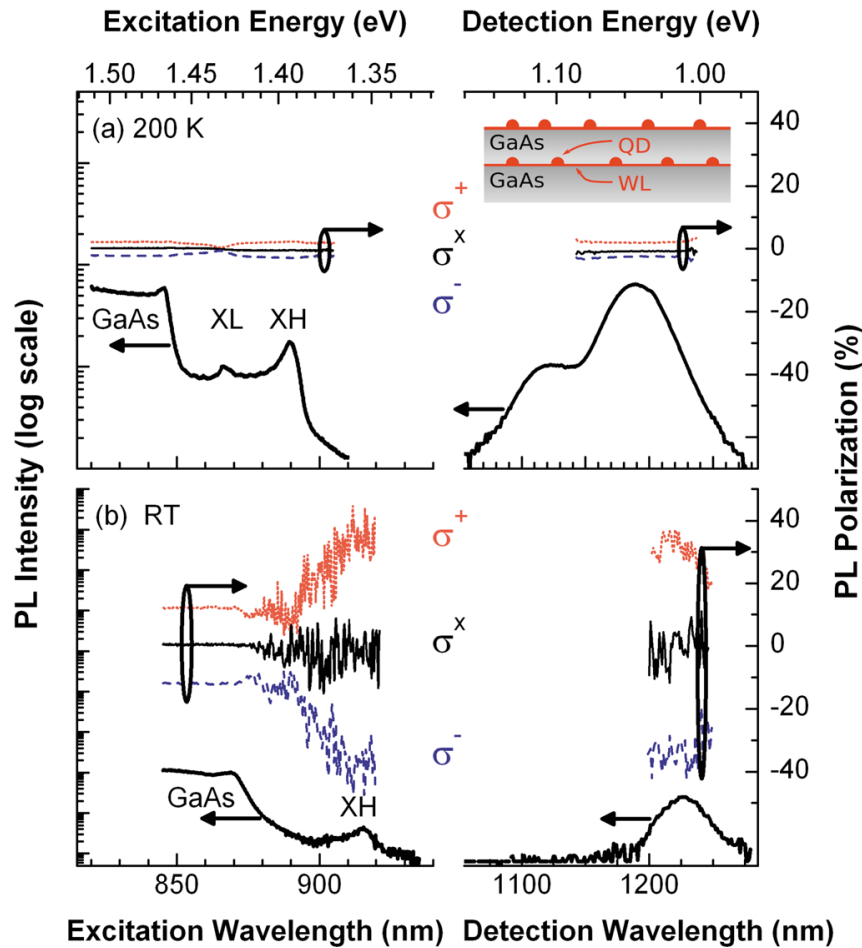

FIG. 1. (Color online) PLE and PL spectra at (a) $200 \mathrm{~K}$ and (b) RT without magnetic field. The PL spectra [the lowest curves on the right panel of (a) and (b)] were taken under excitation in the WL hh band continuum. The PLE spectra [the lowest curves on the left panel of (a) and (b)] were obtained by monitoring the PL intensity at the maximum of the QD ground state PL band. For the polarization spectra, shown by the upper three curves in (a) and (b), the excitation polarization state has been given by $\sigma^{+}, \sigma^{-}$, or $\sigma^{\mathrm{x}}$. For clarity, PLE and PL intensity scale ranges have been adjusted individually for each plot, whereas the polarization scales are identical in al graphs. A sketch of the studied sample structures is shown in the inset. 
the majority of the studied QDs are positively charged with a residual hole. A charged exciton (i.e., a positive trion) is preferably formed in such QDs upon arrival of an electronhole pair created by optical excitation. The light emission from the QDs is hence dominated by the trions. As optical polarization $P$ of the trion is determined by the spin polarization $\rho$ of the electron within the trion, ${ }^{11}$ i.e., $P=-\rho$ when only the heavy-hole (hh) ground state trions are monitored as in our case, it can be employed as an optical spin detector to directly measure spin polarization of optically injected electrons.

PL and PL excitation (PLE) spectra were taken within the temperature range of 100-300 K under excitation from a tunable Ti:sapphire laser. PL was dispersed by a grating monochromator and detected by either a cooled Ge detector or a charge-coupled device camera. Polarization (either of circular $\sigma^{+}, \sigma^{-}$, or linear $\sigma^{\mathrm{x}}$ ) of the excitation beam in optical orientation experiments was controlled by a rotatable quarter-wave plate. PL polarization was analyzed by using either a photoelastic modulator or a quarter-wave plate in conjunction with a fixed linear polarizer. Circular polarization degree of PL is defined by $P=\left(I^{\sigma+}-I^{\sigma-}\right) /\left(I^{\sigma+}+I^{\sigma-}\right)$, where $I^{\sigma+}$ and $I^{\sigma-}$ denote the intensities of $\sigma^{+}$and $\sigma^{-}$polarized PL, respectively. Both excitation and detection direction coincided with the sample growth direction. The measurements in a magnetic field were performed with a split-coil superconducting magnet.

Figure 1 shows representative PLE and PL spectra from the studied QDs at $200 \mathrm{~K}$ and RT. The PL spectra are dominated by the PL emission from the ground state of the trions, where the participating holes are of a hh character due to compressive strain. Similar PL polarization was observed over the whole spectral range of the PL band, which is contributed from QDs with various sizes and chemical compositions. An additional PL band at shorter wavelengths originates from the first excited state as shown by state-filling spectroscopy. ${ }^{12}$ Monitoring the PL from the QD ground state, the PLE spectra cover excitation of carriers in the GaAs barrier and the wetting layer (WL) at the short and long wavelengths, respectively. In the latter case, besides the weaker optical absorption within the WL band continuum, distinct features arising from the WL hh and light-hole (lh) free excitons (marked by $\mathrm{XH}$ and XL, respectively) can also be clearly seen. XH lies at lower energy than XL due to compressive strain. The observed weaker absorption of the $\mathrm{WL}$ as compared to that of the GaAs layer is due to the much lower thickness of the former. Optical selection rules ${ }^{1}$ predict complete spin polarization of photogenerated carriers (before spin relaxation takes place) under circularly polarized excitation from the WL hh band, which we use here for optical spin injection. The created carriers are subsequently injected into the QDs and will, within their lifetime, recombine there. If the injected electrons maintain their spin polarization, the PL from the ground state of the trions should be circularly polarized. For excitation at energies higher than XL, e.g., when both hh and lh bands of the WL are involved, a lower spin polarization of photogenerated carriers is expected as both spin orientations are simultaneously created. ${ }^{13}$ Indeed, the highest PL polarization degree was observed for excitation within the hh part of the WL alone. Under simultaneous excitation from both hh and lh bands in the WL, PL polarization is significantly reduced.

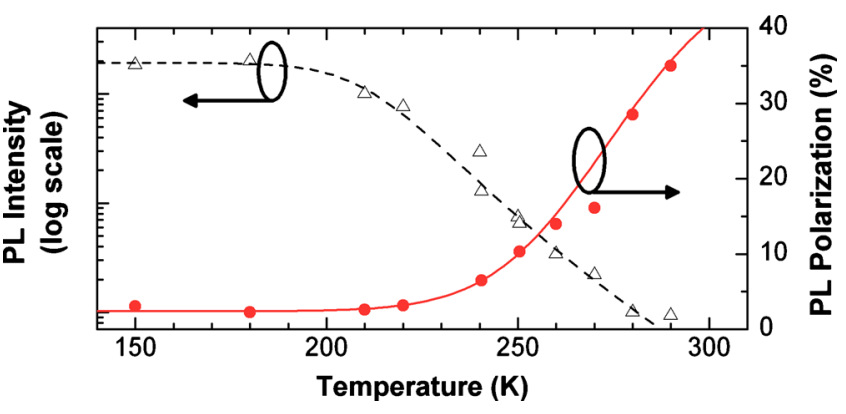

FIG. 2. (Color online) PL intensity (open triangles) and polarization (filled circles) of the trions from the QDs under $\sigma^{+}$excitation in the WL hh band continuum, without magnetic field. The dashed line is a fitting curve based on Eq. (1), yielding an activation energy of $400 \mathrm{meV}$. The solid line is a guide to the eyes.

Common to all regions in the PL and PLE spectra in Fig. 1 is a dramatic increase in PL polarization and thus electron spin polarization of the trion ground state with increasing temperature under $\sigma^{+}$or $\sigma^{-}$excitation. Under $\sigma^{\mathrm{x}}$ excitation, on the other hand, no PL polarization can be detected within the same temperature range. This confirms the spin injection origin of the observed polarization under $\sigma^{+}$or $\sigma^{-}$excitation. A full temperature dependence of optical (and thus electron spin) polarization over the range of $150-300 \mathrm{~K}$ is displayed in Fig. 2, exhibiting a remarkably strong increase in spin polarization degree with increasing temperature. The same trend is found to be true for all studied samples.

Several possible mechanisms could in principle cause the observed thermally activated enhancement of electron spin polarization. One mechanism could be due to a decreasing carrier density in the QD ground state with increasing temperature, which reduces the chance of it being filled by two electrons of opposite spins that would decrease spin polarization. This mechanism can, however, be ruled out here based on our finding that spin polarization is nearly independent of optical excitation power (thus carrier density) over the studied temperature range.

Another mechanism arises from the effect of carrier spin relaxation induced by the hyperfine (hf) interaction between the carriers and nuclear spins in the QDs. Recently, an increase in dynamic nuclear polarization (DNP) with increasing temperature (up to $55 \mathrm{~K}$ ) under optical pumping has been observed, ${ }^{14}$ which can lead to suppression of hf-mediated electron spin relaxation. We can exclude this effect here at high temperatures, based on the following findings. First, rapidly switching excitation between $\sigma^{+}$and $\sigma^{-}$polarization at $50 \mathrm{kHz}$ is not expected to induce significant DNP, as the typical time for build-up of nuclear spin polarization is on the order of millisecond. ${ }^{15}$ Yet, the same PL polarization degree can be obtained under such excitation condition as that observed under cw circularly polarized excitation. Second, the effect of the hf interaction is also characterized by a decrease in spin polarization at a nonzero longitudinal magnetic field in the direction that is opposite to the optically induced Overhauser field such that the effective field acting on the electrons is zero. ${ }^{16}$ As a result, the field-dependent polarization curves should show a symmetric shift of this polarization dip from zero field under $\sigma^{+}$and $\sigma^{-}$excitation because they will lead to oppositely oriented Overhauser fields. The lack of such a characteristic dip in our study, see Fig. 3, leads us to the conclusion that a DNP related effect 


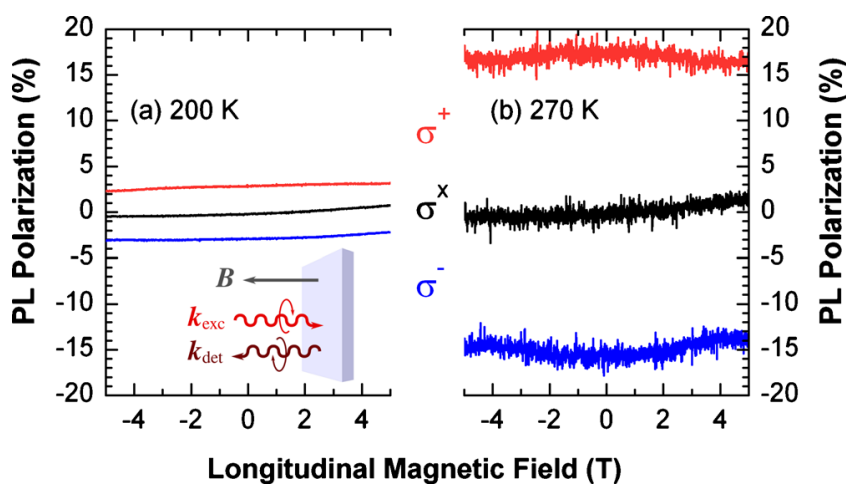

FIG. 3. (Color online) PL polarization as a function of a longitudinal magnetic field at (a) $200 \mathrm{~K}$ and (b) $270 \mathrm{~K}$ under $\sigma^{+}, \sigma^{-}$, and $\sigma^{\mathrm{x}}$ excitation. A weak, linearly changing component of the polarization with magnetic field (more visible under $\sigma^{\mathrm{x}}$ excitation) is ascribed to thermal redistribution between the two Zeeman levels in the QDs. The inset in (a) shows a sketch of the measurement geometry.

cannot be responsible for our observed polarization increase at high temperatures.

We can also rule out the possibility of the recently discovered defect-engineered spin filtering effect as the cause for the observed increase in electron spin polarization with increasing temperature. ${ }^{17}$ This is based on the fact that no substantial difference in PL intensity under linearly and circularly polarized excitation was detected, which is a signature of the defect-engineered spin filtering effect.

Interestingly, we note that the observed increase in spin polarization in all samples is clearly accompanied by a decreasing PL intensity with increasing temperature (see Fig. 2). To shed light on their possible link, temperature dependence of the PL intensity $I(T)$ and the relevant thermal quenching processes were analyzed by the following equation:

$$
I(T)=\frac{I_{0}}{1+\sum_{i} A_{i} e^{-E_{\mathrm{a} i} / k_{\mathrm{B}} T}} .
$$

Here $I_{0}$ is the low-temperature $(10 \mathrm{~K})$ PL intensity value and $k_{\mathrm{B}}$ the Boltzmann constant. $A_{i}$ and $E_{\mathrm{a} i}$ are a constant and activation energy for the $i$ th individual activation process contributing to the observed thermal quenching of the PL intensity, respectively. Our analysis reveals that the PL thermal quenching is dominated by one activation process, with an activation energy of $160-400 \mathrm{meV}$ that varies between the samples. The value is found to lie within the range of the energetic distance between the QD ground state emission and the WL XH for each sample. This suggests that the main mechanism for the observed thermal quenching of the PL close to RT is exciton reemission into the WL, which leads to a shortening of trion lifetime. As electron spin polarization $\rho$ of the trion is a function of trion lifetime $\tau$ and spin relaxation time $\tau_{s}$ according to $\rho \propto\left(1+\tau / \tau_{s}\right)^{-1}$, a shorter trion lifetime can limit electron spin relaxation leading to the observed increase in electron spin and optical polarization.

In conclusion, we have studied optical and spin polarization in InAs/GaAs QD structures up to RT under cw optical spin injection. We found a sharp increase in polarization degree with increasing temperature, reaching $35 \%$ at RT-the highest value reported to date in QDs. This finding also shows that the spin detection efficiency of the studied QDs is at least $35 \%$. The observed increase in optical/spin polarization is suggested to be a result of a shortening of the trion lifetime by thermal activation of excitons into the WL. Our results have thus provided a solid proof for the feasibility and potential of the QD structures as efficient optical/spin source and optical spin detector operating at RT.

${ }^{1}$ Spin Physics in Semiconductors, edited by M. I. Dyakonov (Springer, Berlin, 2008).

${ }^{2}$ I. Žutić, J. Fabian, and S. Das Sarma, Rev. Mod. Phys. 76, 323 (2004).

${ }^{3}$ D. Loss and D. P. DiVincenzo, Phys. Rev. A 57, 120 (1998).

${ }^{4}$ G. Burkard, H. Engel, and D. Loss, Fortschr. Phys. 48, 965 (2000).

${ }^{5}$ H. Gotoh, H. Ando, H. Kamada, A. Chavez-Pirson, and J. Temmyo, Appl. Phys. Lett. 72, 1341 (1998).

${ }^{6}$ E. Tsitsishvili, R. V. Baltz, and H. Kalt, Phys. Rev. B 66, 161405 (2002).

${ }^{7}$ L. M. Woods, T. L. Reinecke, and Y. Lyanda-Geller, Phys. Rev. B 66, 161318 (2002).

${ }^{8}$ A. Tackeuchi, R. Ohtsubo, K. Yamaguchi, M. Murayama, T. Kitamura, T. Kuroda, and T. Takagahara, Appl. Phys. Lett. 84, 3576 (2004).

${ }^{9}$ S. Suraprapapich, S. Panyakeow, and C. W. Tu, Appl. Phys. Lett. 90, 183112 (2007).

${ }^{10}$ S. Suraprapapich, Y. M. Shen, V. A. Odnoblyudov, Y. Fainman, S. Panyakeow, and C. W. Tu, J. Cryst. Growth 301-302, 735 (2007).

${ }^{11}$ A. S. Bracker, E. A. Stinaff, D. Gammon, M. E. Ware, J. G. Tischler, A. Shabaev, A. L. Efros, D. Park, D. Gershoni, V. L. Korenev, and I. A Merkulov, Phys. Rev. Lett. 94, 047402 (2005).

${ }^{12}$ J. Beyer, I. A. Buyanova, S. Suraprapapich, C. W. Tu, and W. Chen, J. Phys.: Conf. Ser. 245, 012044 (2010).

${ }^{13}$ J. Beyer, I. A. Buyanova, S. Suraprapapich, C. W. Tu, and W. M. Chen, Nanotechnology 20, 375401 (2009).

${ }^{14}$ B. Urbaszek, P. Braun, T. Amand, O. Krebs, T. Belhadj, A. Lemaître, P. Voisin, and X. Marie, Phys. Rev. B 76, 201301 (2007).

${ }^{15}$ P. Maletinsky, A. Badolato, and A. Imamoglu, Phys. Rev. Lett. 99, 056804 (2007)

${ }^{16}$ P. Maletinsky, C. W. Lai, A. Badolato, and A. Imamoglu, Phys. Rev. B 75, 035409 (2007).

${ }^{17}$ X. J. Wang, I. A. Buyanova, F. Zhao, D. Lagarde, A. Balocchi, X. Marie, C. W. Tu, J. C. Harmand, and W. M. Chen, Nature Mater. 8, 198 (2009) 